\title{
Siguiendo el modelo sudamericano. Intercambios transnacionales en la continentalización de la FIFA y creación de la UEFA. 1929-1959
}

\author{
Following the "South American model". \\ Transnational exchanges around the continentalization of the FIFA \\ and the creation of UEFA. 1929-1959
}

\section{Philippe Vonnard*}

* Doctor en Educación Física y Ciencias del Deporte por la Universidad de Lausana, Suiza (tesis: «La genèse du football européen», diciembre de 2016). Investigador FNS senior en el proyecto «La fabrique des sports nationaux». Participa activamente en las redes nacionales (Suiza y Francia) e internacionales de investigadores en historia contemporánea.

•philippe.vonnard@unil.ch http://orcid.org/0000-00018564-1509

\section{RECIBIDO: 12.9.2021}

\section{Resumen}

En junio de 1954, 28 asociaciones nacionales de fútbol de toda Europa se reunieron en la ciudad de Basilea (Suiza) para crear una organización continental. Rebautizada en octubre de 1954 como Unión de Asociaciones Europeas de Fútbol (UEFA), en menos de diez años se convirtió en un actor fundamental en el fútbol europeo, un rol protagónico que siguió ocupando en las décadas siguientes. ¿Qué razones llevaron a los dirigentes del fútbol europeo a crear una organización paneuropea como la UEFA en 1954, un tiempo en que Europa estaba dividida por la Guerra Fría? ¿Cuáles fueron las fuentes de inspiración de los promotores de esta organización? Desde una perspectiva de historia global, el artículo propone responder a estas preguntas haciendo énfasis en la influencia que ejerció Sudamérica sobre los promotores de la UEFA. Esta investigación se basa principalmente en documentos encontrados en los archivos de la FIFA y la UEFA; específicamente, en las actas de los Comités Ejecutivo y de Emergencia, los Congresos y la correspondencia. Además, esta documentación se ha cruzado con documentos provenientes de los archivos de algunas asociaciones nacionales de fútbol y 
ejemplares de los periódicos franceses L'Auto, L'Equipe y France Football, que hacen hincapié en el desarrollo del fútbol en el ámbito internacional.

Palabras clave: historia, organización internacional, intercambio continental, deporte.

\section{Abstract}

In June 1954, 28 national football associations from all over Europe met in Basel, Switzerland, to create a continental organization. Renamed as Union of European Football Associations (UEFA) in October 1954, in less than ten years it became a major actor in European football, a leading role which it continued to play in the decades that followed. How can the creation of this organization be explained during the Cold War, when Europeans were divided? What were the main sources of inspiration for the advocates of UEFA? From a globalized perspective, this paper addresses these two questions by focusing on the role played by South American football administrators in this process. This research is primarily based on documents from the FIFA archives, in particular the minutes of both the Executive and Emergency Committees and the Congresses, as well as mail correspondence. These documents have been cross-referenced with other archives of national associations and a careful review of L'Equipe and France Football, newspapers with international scope.

Keywords: history, international organizations, transcontinental exchanges, sport.

\section{Introducción}

En junio de 1954, 28 asociaciones nacionales de fútbol de toda Europa se reunieron en la ciudad de Basilea (Suiza) para crear una organización continental. Rebautizada en octubre de 1954 como Unión de Asociaciones Europeas de Fútbol (UEFA), en menos de diez años se convirtió en un actor fundamental en el fútbol europeo, un rol protagónico que siguió ocupando en las décadas siguientes (Schotté, 2014). ${ }^{1}$

En el contexto del proceso de integración europea que se dio tras la Segunda Guerra Mundial, la creación de la UEFA no fue un hecho aislado. Desde principios de los años

1 Este trabajo es una versión revisada de un artículo escrito en coautoría con Gregory Quin y publicado en 2017 en la revista Sport in Society bajo el título «Did South America foster European football?: Transnational Influences on the Continentalisation of FIFA and the Creation of UEFA, 1926-1959» (Vonnard y Quin, 2017). Asimismo, se inscribe en mi tesis doctoral, que trata la creación de la UEFA, publicada en 2018 con el título L'Europe dans le monde du football. Genèse et formation de l'UEFA (1930-1960). Agradezco a Alejo Levoratti por la relectura de una versión anterior del texto, así como a Bruno Mora y Marcelo Moraes E. Silva por su ayuda en diversas etapas de la investigación. Por último, también agradezco a Johanna Pérez y Luz Romero su trabajo de traducción. 
cincuenta surgieron iniciativas políticas, económicas, científicas y culturales de alcance paneuropeo. El concepto de organización europea (Europe-organisation) del historiador francés Robert Frank (2004) describe perfectamente el desarrollo de una forma «europea» en la creación de organizaciones. La estructura de la UEFA fue, sin embargo, única en ese contexto. A diferencia de la mayoría de las demás organizaciones continentales, la UEFA no excluyó a los países de Europa oriental, venciendo en cierto modo los antagonismos de la Guerra Fría. Además, incluía a los países ibéricos, España y Portugal, que por entonces estaban en la lista negra de las competencias internacionales debido a su gobierno dictatorial. Debido a estas inclusiones, la UEFA constituyó la organización europea más dominante en la década de 1950 (Vonnard, 2018).

Ahora bien, ¿qué razones llevaron a los dirigentes del fútbol europeo a crear una organización paneuropea como la UEFA en 1954? ¿Cuáles fueron las fuentes de inspiración de los primeros promotores de esta organización? Desde una perspectiva de historia global, este artículo propone responder a estas preguntas, por un lado, revelando el papel de los dirigentes sudamericanos en la continentalización de la FIFA y, por el otro, describiendo la manera en que los europeos se reapropiaron del modelo organizativo sudamericano, simbolizado por la existencia de una confederación continental. Salvo un estudio exploratorio de Paul Dietschy (2013), este enfoque no se ha empleado hasta ahora.

Esta investigación se basa principalmente en documentos de los archivos de la FIFA y la UEFA. Específicamente, en las actas de los Comités Ejecutivo y de Emergencia, los Congresos y la correspondencia. Cabe destacar que a partir de la rica correspondencia entre la FIFA y la Conmebol fue posible rastrear parcialmente el desarrollo de la Confederación Sudamericana. ${ }^{2}$ Los ejemplares de las revistas francesas L'Auto, L'Equipe y France Football contribuyeron a llenar los vacíos, ya que estas publicaciones hacen hincapié en el desarrollo del fútbol en el ámbito internacional.

Este artículo comprende el período entre la década de 1920 y finales de la década de 1950. Está organizado cronológicamente y estructurado en tres partes. En la primera se hace un recuento de la presión que ejerció Sudamérica para lograr una mayor influencia en la gobernanza del fútbol mundial, especialmente dentro de los comités de la FIFA. Esta presión en los años de entreguerras generó una ruptura entre los representantes sudamericanos y europeos en la FIFA. La segunda parte subraya el papel que desempeñó la Confederación Sudamericana en la reorganización de la FIFA a principios de la década de 1950. En la tercera sección se analiza brevemente cómo la Confederación Sudamericana sirvió inicialmente de modelo para las actividades de la UEFA.

Sin embargo, debido al material utilizado, no fue posible escribir una «histoire à parts égales», como dice el historiador Romain Bertrand. En este sentido, podría ser realmente interesante utilizar materiales de los archivos de la Confederación Sudamericana, así como de algunas asociaciones nacionales para completar y discutir los documentos europeos sobre las conexiones entre la nueva generación de administradores de la FIFA-UEFA a principios de la década de 1950. 


\section{Intervención sudamericana}

\section{e internacionalización de la FIFA (1930-1945)}

Entre 1915 y 1916 se creó una confederación de asociaciones sudamericanas de fútbol formada por Argentina, Brasil y Uruguay. La legitimidad de esta primera confederación continental de la historia del juego se reforzó cuando, durante la década de 1920, países como Chile, Bolivia y Perú se unieron a la organización. Esta unión fue parte de un esfuerzo contemporáneo más amplio de cooperación continental en torno a la cultura (Dumont, 2012).

La Confederación puso en marcha en 1916 un campeonato para las naciones sudamericanas, la Copa América, el primer torneo de fútbol organizado a escala continental. Así, mientras las selecciones europeas jugaban en campeonatos regionales como la Copa Internacional o la Copa de los Balcanes (Quin, 2013), Sudamérica avanzaba hacia la globalización de este deporte. A pesar de la entrada de varios países no europeos a la FIFA y de algunas decisiones tomadas por su Comité Ejecutivo de abrirse a otros continentes (por ejemplo, la llegada del uruguayo Enrique Buero al Comité Ejecutivo de la FIFA y la creación del cargo de asesor para los continentes fuera de Europa), en el momento de la organización de la primera Copa del Mundo en Uruguay, la pequeña élite dirigente de la FIFA seguía estando conformada en su mayoría por miembros de asociaciones europeas.

El torneo de 1930 en Uruguay marcó un primer cambio en las relaciones entre los líderes sudamericanos y los europeos. Para empezar, los miembros del Comité Ejecutivo de la FIFA, como Rodolphe Seeldrayers o Jules Rimet, quedaron muy impresionados por la organización del torneo, la arquitectura de enormes estadios - el Centenario en Montevideo-, el entusiasmo de los aficionados locales y, por supuesto, la calidad del fútbol de la región. Se hizo evidente que el fútbol ya no era un juego exclusivamente europeo. Sin embargo, la federación sudamericana no estaba muy contenta con la falta de interés de los países europeos por viajar a su hemisferio. A pesar de que la mayoría de los miembros de la FIFA había votado en el Congreso de 1929 que Uruguay fuera la sede de la primera Copa Mundial (FIFA, Congreso, 17 y 18 de mayo de 1929), solo cuatro países europeos (Bélgica, Francia, Rumania y Yugoslavia) participaron en el torneo. Con excepción de las asociaciones británicas que se habían retirado de la FIFA en 1928 (Beck, 2001), los equipos más fuertes de Europa Central (Austria, Hungría, Checoslovaquia) e Italia decidieron no ir, prácticamente boicoteando el evento (Jalabert D’Amado, 2020). Irónicamente, dos semanas antes de la Copa Mundial, los principales países centroeuropeos disputaron en Ginebra (Suiza) un torneo internacional llamado La Coupe des Nations que fue ampliamente cubierto por la prensa europea. 
Esta apatía, unida a la realización de un evento alternativo, generó tensiones entre Sudamérica y Europa. Unos meses antes de la Copa Mundial, el periódico francés Football había anunciado un posible boicot sudamericano contra los equipos europeos que declinaran el viaje («Sud-Américains fâchent», 1930). Esta era una amenaza muy factible dado el favorable ambiente económico de Sudamérica en la época (Díaz Alejandro, 1984) y los estrechos lazos diplomáticos que estaban tejiendo sus gobiernos (Compagnon, 2013). En la década de 1930, las asociaciones sudamericanas exigieron mayor reconocimiento y adelantaron una campaña para tener una cuota de participación dentro del Comité Ejecutivo de la FIFA. Esto llevó a un enfrentamiento con los países europeos y a que solo Brasil y Argentina participaran en la segunda Copa del Mundo, en Italia en 1934.

Como señaló acertadamente Paul Dietschy (2013), el conflicto entre Sudamérica y Europa alcanzó un punto álgido durante la segunda ronda de fútbol en los Juegos Olímpicos de 1936, cuando el jurado del torneo invalidó la victoria de Perú sobre Austria y la Asamblea General de la FIFA, celebrada unas horas después del partido, se negó a revocar esa decisión. Unos meses después, un Congreso Extraordinario de la Confederación Sudamericana decidió intensificar la cooperación interna para posicionarse eficazmente como grupo de presión dentro de la FIFA. Lamentablemente, las diferencias dentro de la Confederación Sudamericana resultaron perjudiciales. Países como Chile y Perú sugirieron retirarse de la FIFA (Schricker, 1936). Sin embargo, la Confederación Sudamericana no parecía estar totalmente de acuerdo sobre este punto. Así, otros países, como Argentina y Brasil, discreparon y consideraron que retirarse de la FIFA era una medida muy extrema. Aunque no se consiguió la unanimidad, al final se propuso concretamente exigir un lugar permanente para Sudamérica en el Comité Ejecutivo de la FIFA.

Pese al conflicto que amenazaba con dividir a la Confederación Sudamericana, los administradores del fútbol europeo tomaron en cuenta sus demandas. Este cambio de actitud se vio propiciado por la creciente importancia de los países no europeos dentro de la FIFA. A ello se sumó la creación de una confederación en Centroamérica a finales de la década de 1930 por parte de Héctor Beeche, que se solidarizó con Sudamérica. En 1939, el presidente Jules Rimet viajó a Sudamérica y se reunió con los administradores del fútbol para tratar de disminuir la tensión (Schricker, 1939). Si bien esta medida no fue más que un apaciguamiento a corto plazo, la estructura de la FIFA sufrió importantes cambios en la década siguiente debido tanto a la influencia de la Segunda Guerra Mundial como al fortalecimiento de una voz continental sudamericana en el fútbol.

La FIFA funcionó de forma reducida durante la Segunda Guerra Mundial (Vonnard y Quin, 2019), especialmente después de 1942. Las emergencias y restricciones de los tiempos de guerra dificultaron que el secretario Ivo Schricker mantuviera correspon- 
dencia con las federaciones sudamericanas. Como si fuera poco, el miembro sudamericano del Comité Ejecutivo, Luis Dupuy, tuvo que regresar a su país en 1943 (Schricker, 1943). Fue una pérdida importante para Schricker porque Dupuy era su enlace con Sudamérica y también un compañero de confianza. Más aún, Dupuy era un funcionario emprendedor, siempre dispuesto a tener discusiones significativas en lugar de limitarse a disentir con la FIFA. Mientras esto ocurría en Europa, la Confederación Sudamericana adoptó medidas decisivas para fortalecer los consensos entre sus miembros y construir una voz propia dentro de la FIFA. Luis Valenzuela, el nuevo presidente de la Confederación, nombrado en 1939, era a todas luces un firme defensor del fútbol sudamericano. Defendió su posición dentro de la FIFA durante 15 años e intentó dar más poder a su Confederación. Bajo su influencia se crearon nuevas relaciones entre las asociaciones del continente. En 1941 se dio un paso importante cuando Argentina regresó a la organización. Luego, en 1942, se discutió la idea de celebrar un Día del Fútbol Sudamericano para fortalecer simbólicamente la fraternidad entre las asociaciones. Hay que señalar que estas acciones coinciden con un nuevo impulso del panamericanismo, consolidado ya en diferentes ámbitos durante la década de 1930 (véase, por ejemplo, Di Liscia y Álvarez, 2019), que contribuyó a estrechar lazos dentro del continente sudamericano (Compagnon, 2015, pp. 590-592).

En este contexto, la Confederación decidió exigir más autonomía dentro de la FIFA. Primero, en 1941, expresó su interés por organizar la siguiente Copa del Mundo. Luego decidió enviar a Zúrich solo la mitad del monto de los ingresos financieros de los partidos internacionales disputados en Sudamérica (Homburg, 2008). En una época en la que el fútbol de Europa, devastado por la guerra, apenas daba beneficios, el secretario de la FIFA, Ivo Schricker, tomó a mal esta decisión. Finalmente, en 1945, Agustín Matienzo, el nuevo delegado de la Confederación Sudamericana en el Comité Ejecutivo de la FIFA envió una carta en la que proponía organizar el siguiente Congreso de la FIFA en 1946 en Montevideo. En su carta señaló que el Comité Ejecutivo de la FIFA era entonces ilegítimo, ya que su mandato había concluido en 1942 (Matienzo, 1945). Esta acción muestra cómo Sudamérica desafiaba abiertamente el control exclusivo de la FIFA sobre la gobernanza internacional del fútbol.

\section{El impacto de Sudamérica en la continentalización de la FIFA (1946-1951)}

La tensión entre Europa y Sudamérica aumentó a medida que en los años siguientes los miembros sudamericanos pedían más legitimidad dentro de la FIFA. Los sudamericanos querían descentralizar la autoridad exclusiva de la FIFA sobre el fútbol mundial 
otorgando más poder e influencia a cada uno de los segmentos continentales del órgano directivo. Esta situación reflejaba el cambio de paradigma en la cooperación mundial tras la Segunda Guerra. Como ha demostrado Eric Helleiner (2014), este cambio se caracterizó por la alianza entre países no europeos y por la creación de nuevas organizaciones internacionales que incluían a los Estados Unidos y a países de Asia o Sudamérica. Después de la guerra, las potencias coloniales europeas perdieron su superioridad política y la mayor influencia mundial empezaron a ejercerla los Estados Unidos de América y la Unión Soviética.

Para ganar efectividad dentro de la FIFA, Sudamérica puso en práctica una idea discutida en la década del treinta: la de crear una organización panamericana. Incluida por primera vez en los nuevos estatutos de la Confederación Sudamericana en 1941, la idea tomó forma concreta en 1946, en la ciudad de Barranquilla (Colombia). Para 1948 - año en que se creó la Organización de los Estados Americanos- la organización panamericana contaba con 19 miembros (FIFA, 1948). Si todos los miembros hubieran perseguido un mismo objetivo en los años de entreguerras, se podría haber creado un poderoso bloque dentro de la FIFA para ejercer una presión sin precedentes sobre los países europeos. El momento era todavía mejor que en los años treinta. Las consecuencias geopolíticas de la guerra, especialmente la controversia sobre el estatus de Alemania (Wahlig, 2010) e Italia (Sbetti, 2016), eran motivo de preocupación. Al mismo tiempo, la expansión del comunismo en Europa central creó una fisura entre las asociaciones de fútbol de Europa oriental y occidental. En el contexto de los fuertes enfrentamientos Oriente-Occidente, la participación en las organizaciones deportivas fue crucial para la Unión Soviética (Dufraisse, 2018) y los Estados Unidos (Rider, 2017). Para ambos países el deporte se convirtió en un escenario de lucha simbólica entre el capitalismo y el comunismo. De esta manera, la Guerra Fría agudizó la ruptura entre las asociaciones del fútbol europeo, hasta el punto de que desde mediados de 1949 hasta mediados de 1952 no se disputaron partidos entre los países europeos a un lado y otro de la Cortina de Hierro y se suspendieron competencias como la Copa Internacional («Coupe internationale», 1949).

Adicionalmente, para hacer operativa la descentralización de la FIFA se consideró necesaria su reestructura en relación con el desarrollo del propio fútbol. De hecho, muchos de los nuevos países independientes de Centroamérica y Asia se afiliaron a la FIFA durante las décadas de 1940 y 1950, lo que representó una expansión del mundo del fútbol. De unos 30 miembros a mediados de 1930 se pasó a más de 70 a principios de la década del cincuenta y se llegó a 88 en 1955. El fútbol se extendió a lo largo y ancho del mundo a medida que el transporte aéreo aumentó el número de intercambios internacionales, como la circulación de jugadores y entrenadores (Taylor, 2006) y la realización de partidos entre selecciones nacionales y clubes y torneos intercontinentales (por ejemplo, la Copa Río, que empezó en 1951). 
El incremento en el número de miembros se explica también por el contexto de los procesos de descolonización que atravesaban varias naciones. El fútbol $-\mathrm{y}$, en general, el deporte - representaba para los nacientes Estados-nación un medio para fomentar la unidad nacional, para afianzar las identidades nacionales independientes y para ser reconocidos por la comunidad internacional. Por eso los países que aún estaban colonizados, como Costa de Oro, o que se resistían a la dominación colonial, como Vietnam, solicitaron ser miembros de la FIFA a principios de la década de 1950. Sin embargo, estos nuevos países querían algo más que ser miembros: querían también un espacio en la dirección de la FIFA. Por ejemplo, durante una reunión del Comité Ejecutivo en junio de 1950, Costa de Oro le pidió a la FIFA su apoyo para la creación de una nueva Asociación Africana de Fútbol Unificada, formada por las asociaciones de Sudáfrica, Costa de Marfil, Costa de Oro, Nigeria, Sierra Leona y Togo. El objetivo de esta asociación era «crear vínculos más estrechos entre las asociaciones africanas para intercambiar puntos de vista, organizar el pago de las contribuciones de los miembros, programar partidos regulares entre colonias y realizar una competencia anual entre colonias» (FIFA, 1950a).

Por último, debido a problemas derivados de las eliminatorias del Mundial de 1950, se argumentó que la gestión de un deporte tan extendido a escala mundial era demasiado complicada para estar en manos de un solo ente administrativo. La distancia entre la sede de la FIFA y numerosos miembros de la Federación —distancia tanto en sentido literal como figurado - alimentó la idea de que era imperativo descentralizar la FIFA para mejorar la gestión y la práctica del fútbol en todo el mundo.

Todos estos hechos legitimaron todavía más las exigencias sudamericanas. En el

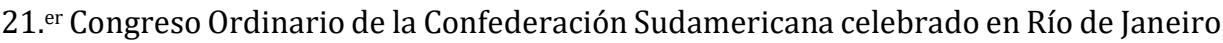
en mayo de 1949, los delegados discutieron una posible reforma de los estatutos de la FIFA. Fundamentalmente pretendían que la FIFA reconociera a Sudamérica asignándole cargos adicionales en el Comité Ejecutivo (FIFA, 1949). El único cargo que tenían asignado parecía estar bajo amenaza, pues el número de miembros de la FIFA no dejaba de aumentar. En efecto, dado que los miembros del Comité Ejecutivo se elegían por votación en los Congresos, cuya asistencia prácticamente se había duplicado en los últimos años, la Confederación Sudamericana temía que una distribución más global del poder redujera la autoridad que reclamaba. Al final, los miembros sudamericanos enviaron dos propuestas que se discutieron durante el Congreso de la FIFA de 1950, el primero que se organizó fuera de Europa, en Río de Janeiro durante la Copa del Mundo -la cual impresionó a los observadores europeos, especialmente por la creación del estadio más grande del mundo: el Maracanã (Astruc, 2020)—. Los objetivos de Sudamérica eran claros: dividir la FIFA en grupos continentales que controlaran y gestionaran sus propias esferas futbolísticas, en lugar de solo elegir representantes en los principales comités de la FIFA (FIFA, 1950b). Pese a los desacuerdos existentes entre 
algunos países sudamericanos - por ejemplo, Argentina no participó en la Copa del Mundo celebrada en Brasil en 1950-, los documentos recogidos en la FIFA muestran que a finales de los años cuarenta surgió un consenso entre las asociaciones sudamericanas de football. ${ }^{3}$

Fue entonces evidente la necesidad de un cambio en la organización de la FIFA, y algunos administradores del fútbol europeo comenzaron a plantearse una revisión de los estatutos. El Congreso de 1950 encargó una investigación especial para analizar la coyuntura y proponer posibles medidas. A pesar de la evidente preeminencia de los delegados europeos en esta nueva comisión (solo dos de los siete delegados procedían de las asociaciones sudamericanas), existía la esperanza de un cambio. De hecho, la idea de reorganizar la FIFA contó con el apoyo de una nueva generación de administradores del fútbol europeo, influidos por la organización y el éxito del fútbol en Sudamérica. Se trataba de Ottorino Barassi (Italia), Stanley Rous (Inglaterra) y Ernst Thommen (Suiza), quienes se convirtieron en las personas más importantes de la FIFA a principios de la década de los cincuenta, tras la retirada de Schricker. El nuevo secretario general, Kurt Gassmann, era colega de Thommen desde finales de los años treinta, antiguo secretario de la federación suiza, y gozaba de una gran credibilidad. Para Barassi, Rous y Thommen, había llegado el momento de considerar la posibilidad de crear organizaciones continentales dentro de la FIFA, siguiendo el modelo de las confederaciones sudamericanas. En 1952 Barassi tomó la iniciativa y se dirigió por carta a varias asociaciones europeas para comunicarles la idea de crear una organización europea (Barassi, s.f.). Estos nuevos administradores del fútbol se desvincularon de la antigua élite que se estaba retirando gradualmente de la FIFA y tomaron impulso cuando Jules Rimet, cuya perspectiva limitó siempre la implementación de los cambios propuestos, finalmente se jubiló en 1954 (Quin y Vonnard, 2014).

A pesar de estos avances, la nueva élite no pretendía una reorganización inmediata de la Federación, sino una transición gradual. Para ello acordó una nueva versión de la FIFA dividida en grupos continentales con poder moderado. Teniendo como punto de referencia el torneo de la Copa América que puso en marcha la Confederación Sudamericana, se lanzaría también un torneo paneuropeo, idea previamente discutida durante el período de entreguerras en el marco del proyecto de creación de una federación continental europea. Barassi, al haber viajado muchas veces a Sudamérica por trabajo,

Cabe destacar que son dos las propuestas que llegan de Sudamérica (una proveniente de Argentina, Paraguay y Uruguay, la otra de Chile, Bolivia y Perú), lo que demuestra que hay discrepancias entre las asociaciones del continente sobre el modo de reformar la FIFA. Sin embargo, ambos proyectos coinciden en la necesidad de restructurar la organización internacional y de dar más espacio a los países sudamericanos en la gobernanza de la Federación Internacional. 
probablemente tuvo la oportunidad de presenciar directamente la organización del fútbol y de conversar con los presidentes y secretarios de las asociaciones de fútbol, lo cual influyó profundamente en su decisión de replicar el modelo sudamericano.

Sin embargo, no todos los directivos europeos compartían estas ideas. Por ello, durante las reuniones de las asociaciones europeas en Zúrich y París, celebradas en mayo y junio de 1952 respectivamente, bajo la presidencia de Barassi («Treize ont terminé», 1952), la idea de crear un grupo europeo fue rápidamente descartada. Estas sesiones permitieron discutir otras propuestas de reorganización de la FIFA, tema que debería debatirse en el siguiente Congreso de la FIFA en Helsinki unas semanas más tarde (FIFA, 1952). Es importante señalar que ningún país de Europa oriental, ni siquiera Yugoslavia, que era un país no alineado y protegía a Europa occidental del bloque soviético (Svetozar, 2011), participó en las reuniones. Sin duda, esta falta de participación frenó la inclusión de la Unión Soviética y sus países satélites en esta nueva organización. La Guerra Fría seguía restringiendo los intercambios en el fútbol continental. No obstante, en el Congreso de la FIFA celebrado en Helsinki en 1952 se dio un paso hacia la creación de una organización europea. Una vez más, no es posible entender esta situación sin tener en cuenta el importante papel desempeñado por los líderes sudamericanos.

\section{Siguiendo el modelo sudamericano. La creación de la UEFA (1952-1959)}

El debate sobre la descentralización de la FIFA continuó en Helsinki. Varios representantes sudamericanos iniciaron largas discusiones sobre cuestiones reglamentarias (como elegir más de una lengua oficial, el manejo de la correspondencia) que parecían accesorias a los europeos. En contraste con años anteriores, en los que los delegados sudamericanos presionaban a los europeos para que cedieran terreno, por primera vez ambos mostraron interés en la negociación de un mismo reglamento. Se decidió informar de la decisión final en un Congreso Extraordinario que se celebraría en 1953.

En cuanto al debate en el Congreso, José Crahay - otro delegado de la nueva generación y uno de los primeros promotores de la idea de la Asociación Europea de Fútbol- escribió en el libro conmemorativo de la UEFA de 1979:

Nos dimos cuenta de que cada uno de los puntos del orden del día [había] sido estudiado a fondo y que los delegados [sudamericanos] [habían] sido efectivamente nombrados para defender posiciones frecuentemente diferentes de las apoyadas por el Comité Ejecutivo de la FIFA. (Rothenbühler, 1979) 
La formación de un bloque sudamericano en Helsinki impactó a varios delegados europeos y los convenció de crear un grupo de presión similar en Europa como contrapeso. Hacia mediados de 1952 comenzó a materializarse la idea de crear un agrupamiento europeo, idea en la que la actitud de los miembros del fútbol sudamericano había tenido una importante influencia.

En los meses siguientes, la idea de crear una organización unificada europea se debatió en múltiples niveles, pero había que resolver una serie de problemas antes de que pudiera concretarse. Una vez más, la actuación de los dirigentes sudamericanos obligó a las asociaciones europeas a cambiar su estrategia. En esta ocasión, los sudamericanos abogaron por que los europeos tuvieran puestos en los futuros Comités Ejecutivos. Aunque esta propuesta pudo parecer paradójica a primera vista, fue una excelente táctica para refrendar la idea de una continentalización en el seno de la FIFA, y así ayudar a Sudamérica a conseguir un lugar en el Comité Ejecutivo junto a Europa. Al mismo tiempo, les permitió mejorar sus relaciones con los miembros europeos tras los crudos intercambios del Congreso de 1952. El tema se planteó en la sesión de la Comisión celebrada los días 6 y 7 de marzo de 1953, y fue allí donde dos miembros sudamericanos solicitaron formalmente reservar puestos para Europa, «de una vez por todas», en el Comité Ejecutivo. Si bien la propuesta siguió tropezando con cierta oposición, fue un verdadero avance que se materializaría a lo largo de los siguientes meses.

En junio, Barassi y Rous se reunieron en casa de Thommen, en Basilea, para discutir la cuestión de los puestos en el Comité Ejecutivo para los europeos. Estaba presente también Ebbe Schwartz, el delegado de las asociaciones escandinavas. La presencia de estas cuatro importantes figuras del fútbol europeo evidencia que la idea de una organización europea estaba en marcha. En Basilea no se tomó ninguna decisión, pero se concertó una nueva reunión con todas las asociaciones europeas antes del siguiente Congreso. Dicha reunión se celebró dos días antes del Congreso Extraordinario de la FIFA («Mais dès Aujourd'hui», 1953). Pese a las dificultades iniciales para conceptualizar un frente unido de las naciones futboleras de Europa, el acuerdo estaba a punto de producirse. De hecho, las asociaciones europeas ya habían anticipado un gran cambio en la estructura de la FIFA y estaban preparadas para adaptarse a él. Por primera vez en la historia de la FIFA, los delegados europeos hablaron en nombre de un "grupo de asociaciones europeas» (FIFA, 1953a). En la reunión se entablaron varias discusiones, y por primera vez con asociaciones procedentes del otro lado de la Cortina de Hierro. Las proactivas asociaciones sudamericanas lograron un compromiso con las asociaciones europeas en la tarde del segundo día del Congreso. El último día, Thommen aprobó la resolución de destinar dos vicepresidencias y cuatro puestos a los europeos en el Comité Ejecutivo (FIFA, 1953a). Esto significaba que los directivos del fútbol europeo finalmente habían aceptado las recomendaciones sudamericanas. 
Las ideas fueron aceptadas por la mayoría de los participantes en el Congreso, con excepción de las asociaciones del bloque soviético, que se opusieron a la reorganización propuesta. El nuevo artículo 17 de los estatutos de la FIFA contemplaba la creación de un grupo europeo. Su quinto párrafo, que debía implementarse durante la primera parte de 1954, estipulaba que «los miembros del Comité Ejecutivo (vicepresidente y miembros) [serían] electos por los grupos de las asociaciones nacionales» (FIFA, 1953b). Teniendo en cuenta el contexto de la Guerra Fría en el que se tomó esta decisión, no fue fácil reunir a todas las asociaciones europeas de fútbol. Por lo demás, si las asociaciones europeas encontraban finalmente un acuerdo para crear una organización, ¿deberían realizar un grupo dedicado únicamente a elegir a los miembros europeos en el Comité Ejecutivo de la FIFA o llevar a cabo algo más ambicioso que potenciara las alianzas entre las federaciones europeas?

Tras nuevas discusiones, una asamblea celebrada en Basilea en junio de 1954 con 25 asociaciones europeas (en realidad 27, porque dos estaban representados por otros) acordó finalmente la creación de un nuevo grupo europeo. Las actividades de los miembros del bloque soviético no se documentaron por separado, pero probablemente entendieron que era necesario unirse al nuevo grupo europeo para que ellos, un pequeño grupo de diez asociaciones, fueran tomados en serio. Además, la transición política en la Unión Soviética tras la muerte de Stalin (Rey, 2005) aumentó las posibilidades de intercambio deportivo entre la Europa oriental y la occidental. Al finalizar la guerra de Corea (1953), la conferencia de Berlín (1954), en la que se reunieron los ministros de Asuntos Exteriores de Estados Unidos, Gran Bretaña, Francia y la Unión Soviética por primera vez desde 1948, puso de manifiesto el deseo de iniciar nuevos diálogos entre países a ambos lados de la Cortina de Hierro. Dado que el fútbol ya había dado pasos en esta dirección, especialmente con la reanudación de la Copa Internacional, la creación de un grupo europeo sería una intervención oportuna para conectar culturalmente a los países. Aunque ninguna organización de Europa occidental había conseguido cruzar la Cortina de Hierro, los dos bloques estaban de hecho vinculados de más formas que las que se reconocían en aquella época (véase, por ejemplo, Hochscherf et al., 2010).

Durante su primer mes, la organización, rebautizada como UEFA en octubre de 1954, apenas era una voz acreditada de la administración del fútbol. Sin embargo, algunos miembros, especialmente el presidente Ebbe Schwartz, querían una rápida consolidación de la organización. Un primer paso fue poner en marcha la Copa de Clubes Campeones de Europa, una idea propuesta por primera vez por Gabriel Hanot, periodista del diario parisino L'Equipe. Este campeonato, celebrado en la temporada 1955-1956, dio mayor legitimidad y planteó nuevos desafíos a la organización europea (Vonnard, 2014). 
Una opción para desarrollar la organización europea era inspirarse en las organizaciones existentes, principalmente la Confederación Sudamericana. Desde esta perspectiva, una posibilidad era pedir a la FIFA que remitiera la mitad del porcentaje (2\%), tal y como ganaba la Confederación Sudamericana, de los partidos jugados entre países europeos. Las comunicaciones entre la UEFA y la FIFA muestran que este tema se discutió durante un año y provocó tensiones entre ambas organizaciones. Finalmente, con la mediación de Ernst Thommen, vicepresidente de la FIFA y presidente de su Comité Financiero en ese momento, la FIFA decidió conceder a la UEFA (y a otras confederaciones, como la asiática, creada en 1956) el mismo derecho que a la Confederación Sudamericana (Vonnard, 2020).

Por otro lado, basándose en el modelo de la Copa América, la idea de un Campeonato Europeo - rechazada por la mayoría de las asociaciones en 1955 - se volvió a discutir a partir de 1956-1957 (Diestchy, 2017). Algunos dirigentes como Gustav Sebes (Hungría), el hijo de Henri Delaunay — quien había defendido con ahínco esta idea desde los años treinta- y Pierre, el nuevo secretario de la UEFA (que sucedió a su padre en 1955), se mostraron partidarios de crear ese campeonato. Ellos, junto con Ebbe Schwartz y José Crahray, consideraron que era importante desarrollar la estructura de la UEFA, así como crear torneos de fútbol y una mayor conexión entre las asociaciones europeas. Tras numerosas discusiones en el Congreso de la UEFA de 1957 y 1958 (UEFA, 1957, 1958), se decidió crear un torneo europeo, que empezó en 1959 (la fase final tuvo lugar en 1960).

De este modo, entre 1954 y 1958, la Confederación Sudamericana influyó directamente en las decisiones tomadas por los administradores del fútbol europeo que condujeron a la creación de la UEFA y de la Copa Europea de Naciones. A finales de la década, la UEFA empezó a ser un actor importante en la FIFA como representante del fútbol europeo y una poderosa contraparte de la Confederación Sudamericana. Estos nuevos actores del fútbol mundial auguraban una nueva era en la historia de la FIFA.

\section{Conclusión}

Este artículo explora la influencia de la Confederación Sudamericana de Fútbol en la creación de la UEFA. Como se indica en la introducción, se basa principalmente en documentos recopilados en Europa, razón por la cual tiene limitaciones en cuanto a la información recopilada y ofrece una visión predominantemente eurocéntrica de los acontecimientos. Sin embargo, el estudio tiene el mérito de proponer un primer paso hacia la elaboración de una historia de las relaciones intercontinentales en el fútbol, así como hacia una mejor comprensión de la continentalización de la FIFA, iniciada en los años cincuenta. De igual forma, propone descentralizar la historia de la 
cooperación europea subrayando la influencia sudamericana en la actuación de los dirigentes europeos.

La investigación ha demostrado que la Confederación Sudamericana influyó en este proceso de tres maneras.

En primer lugar, en su esfuerzo por obtener un mayor reconocimiento dentro de la FIFA, los dirigentes sudamericanos desempeñaron un papel clave en la reorganización que tuvo lugar dentro de la Federación a principios de la década de 1950. Esto desembocó en su continentalización y, con ello, abrió la puerta a la creación de la UEFA.

Una segunda manera en la que la Confederación Sudamericana influyó en la creación de la UEFA tiene que ver con la reflexión que suscitó en los europeos la creciente cohesión de los líderes sudamericanos durante los congresos de la FIFA. La falta de cohesión europea ante lo que se percibía como un bloque sudamericano llevó a una nueva generación de dirigentes europeos (encabezados por el trío Barassi, Rous y Thommen) a desarrollar a la idea de formar una organización del continente europeo a principios de los años cincuenta.

En tercer lugar, si bien en un principio el objetivo de una organización europea era preservar la hegemonía de Europa dentro de la FIFA, pronto se orientó hacia el desarrollo del fútbol europeo. En este sentido, la Confederación Sudamericana sirvió sin duda como modelo. Fue sobre la base de algunas de sus prerrogativas, como la de recibir un porcentaje de la FIFA de los partidos disputados entre sus asociaciones miembros y la creación de un campeonato continental en Sudamérica, que la organización europea consiguió evolucionar y asegurar así una relativa legitimidad.

Por último, es importante tener en cuenta lo que historiadores especializados en historia global han llamado el efecto retorno. Después de haber sido influido por el modelo sudamericano, a partir de mediados de la década de 1950, la UEFA comenzó a ser percibida como un modelo para los administradores de Asia, África e incluso la misma Sudamérica en materia de gobernanza del fútbol. A finales de esa década, Sudamérica siguió el ejemplo europeo al poner en marcha un campeonato de fútbol de clubes, la Copa Libertadores. Como ya ha señalado Christiane Eisenberg (2011), el conocimiento de las conexiones transcontinentales podría ser muy útil para comprender el desarrollo de las estructuras futbolísticas en todo el mundo y, de forma general, la persistencia de la hegemonía europea en el juego del pueblo.

\section{Referencias bibliográficas}

Astruc, C. (2020). Beyond the Maracanazo: The World Cup, diplomacy and the international exposure of Brazilian football in 1950. Soccer \& Society, 21(8), 861-875. 
Barassi, O. (s.f.). [Anexo de una carta a R. Seeldrayers, 1952] (Folio: Réorganisation 5053). Archivo de la FIFA, Zúrich.

Beck, P. J. (2001). ¿Going to war, peaceful co-existence or virtual membership? British football and FIFA, 1928-46. The International Journal of The History of Sport, 17(1), 113-134.

Compagnon, 0. (2013). L'adieu à l'Europe: L'Amérique latine et la Grande Guerre. París: Fayard.

Compagnon, 0. (2015). ¿En marge de la Guerre? Les Amériques Latines. En A. Aglan y R. Frank (dirs.), La guerre-mond: Tome premier (pp. 565-598). París: Gallimard.

¿La Coupe internationale a-t-elle vécu? L'Italie n'aura pas terminé son programme l'an prochain (1949, 19 de abril). France Football.

Di Liscia, M. S., y Álvarez, A. C. (2019). La Cruz Roja: El panamericanismo y la salud en el período de entreguerras. Salud Colectiva, 15. doi: 10.18294/sc.2019.2116.

Díaz Alejandro, C. F. (1984). The 1940s in Latin America. En M. Syrquin, L. Taylor y L. E. Westphal (eds.), Economic structure and performance (pp. 341-362). Nueva York: Harcourt.

Dietschy, P. (2013). Making football global? FIFA, Europe, and the non-European football world, 1912-74. Journal of Global History, 8(2), 279-298.

Dietschy, P. (2017). L'Euro de l'européisme à la commercialisation de la nation. Pôle Sud, 47(2), 25-39.

Dufraisse, S. (2018). Les héros du sport: Une histoire des champions soviétiques (années 1930-années 1980). Ceyzérieu: Champ Vallon.

Dumont, J. (2012). De la coopération intellectuelle à la diplomatie culturelle: Le parcours du Brésil dans l'entre-deux-guerres. Caravelle: Cahiers du Monde Hispanique et Luso-Brésilien, 99, 217-238.

Eisenberg, C. (2011). Towards a new history of european sport? European Review, $19(4), 617-622$.

FIFA. (1948, 30 de marzo). Nómina de asociaciones afiliadas a la Confederación Pan-Americana de Football (Folio: Correspondance avec la CONMEBOL: 1941-1961). Archivo de la FIFA, Zúrich.

FIFA. (1949, marzo-mayo). Congreso de la Confederación Sudamericana (Folio: Correspondance avec la CONMEBOL: 1941-1961). Archivo de la FIFA, Zúrich.

FIFA. (1950a, 22-23 de junio). Congreso (Folio: XXV-XXVII Congrès Ordinaire: 19461952). Archivo de la FIFA, Zúrich.

FIFA. (1950b). Anexo al orden del día del Congreso de la FIFA de 1950 (Folio: XXV-XXVII congrès ordinaire: 1946-1952). Archivo de la FIFA, Zúrich.

FIFA. (1952, 20-23 de julio). Congreso (Folio: XXV-XXVII Congrès Ordinaire: 19461952). Archivo de la FIFA, Zúrich. 
FIFA. (1953a, 14-15 de noviembre). Congreso (Folio: XXIX-XXX Congrès Ordinaire: 1953-1959). Archivo de la FIFA, Zúrich.

FIFA. (1953b). Estatutos de la FIFA (Folio: Statuts, 1904-1981). Archivo de la FIFA, Zúrich. Frank, R. (2004). Les débats sur l'élargissement de l'Europe avant l'Élargissement. En G. Pécout (dir.), Penser les frontières de l'Europe du XIXe au XXe siècle: Élargissement et union. Approches historiques (pp. 179-196). París: PUF.

Helleiner, E. (2014). The forgotten foundations of Bretton Woods: International development and the making of the postwar order. Nueva York: Cornell University.

Hochscherf, T., Laucht, C., y Plowman A. (2010). Divided, but not disconnected: German experiences of the Cold War. Nueva York: Berghahn Books.

Homburg, H. (2008). Financing world football: A business history of the Fédération Internationale de Football Association. Zeitschrift für Unternehmensgeschichte, 53(1), 33-69.

Jalabert D'Amado, L. (2020). Montevideo 1930: Reassessing the selection of the first World Cup host. Soccer \& Society, 21(8), 848-860.

Mais dès Aujourd'hui au Congrès de la FIFA joute oratoire Europe-Amérique latine (1953, 14-15 de noviembre). L'Equipe.

Matienzo, A. (1945, 23 de julio). [Carta a I. Schricker] (Folio: FIFA, Rapport d'activité durant la guerre). Archivo de la FIFA, Zúrich.

Quin, G. (2013). La Coupe de l’Europe Centrale (1927-1938), une compétition internationale oubliée?. Stadion: Revue Internationale d'Histoire du Sport, 37 (2), 285-304.

Quin, G., y Vonnard, P. (2014). La "Présidence inamovible" du football. Jules Rimet (1873-1956). En E. Bayle (dir.), Les grands dirigeants du sport. 23 portraits et stratégies de management (pp. 25-43). Bruselas: De Boeck.

Rey, M.-P. (2005). Le retour à l'Europe? Les décideurs soviétiques face à l'intégration européenne, 1957-1991. Journal of European Integration History, 11(1), 7-27.

Rider, T. (2017). Cold war games: Propaganda, the Olympics, and U.S. foreign policy. Champaign: University of Illinois Press.

Rothenbühler, R. (1979). Les 25 ans de l'UEFA, Berna: UEFA.

Sbetti, N. (2016). The quest for legitimacy: The road to redemption for Italian football in Europe after the Second World War (1943-1949). En P. Vonnard, G. Quin y N. Bancel (eds.), Building Europe with a ball: Turning points in the europeanisation of football (1905-1995) (pp. 101-119). Oxford: Peter Lang.

Schotté, M. (2014). La structuration du football professionnel européen: Les fondements sociaux de la prévalence de la "spécificité sportive". Revue Française de Socio-Economie, 13(1), 85-106.

Schricker, I. (1936, 21 de noviembre). [Carta al Comité Ejecutivo] (Folio: Comité Exécutif: 1935-1936). Archivo de la FIFA, Zúrich. 
Schricker, I. (1939, 21 de abril). [Carta al Comité Ejecutivo] (Folio: Comité Exécutif: 1939). Archivo de la FIFA, Zúrich.

Schricker, I. (1943, 17 de marzo). [Carta a E. Borrero] (Folio: Correspondance avec la CONMEBOL: 1941-1961). Archivo de la FIFA, Zúrich.

Les Sud-Américains se fâchent (1930, 30 de mayo). Football.

Svetozar, R. (2011). Yugoslavia and the Soviet Union in the early Cold War: Reconciliation, comradeship, confrontation, 1953-1957. Londres: Routledge.

Taylor, M. (2006). Global players? Football, migration and globalization, c. 1930-2000. Historical Social Research, 31(1), 7-30.

Les treize ont terminé leur travaux et nommé leur Bureau (1952, 29 de marzo). $L^{\prime} E$ quipe.

UEFA (1957, 28-29 de junio). Congresso (Folio: RM00005984. Congresso 1954-1994). Archivo de la UEFA, Nyon.

UEFA (1958, 4 de junio). Congresso (Folio: RM00005984: Congresso 1954-1994). Archivo de la UEFA, Nyon.

Vonnard, P. (2014). A competition that shook European football: The origins of the European champion clubs' cup, 1954-1955. Sport in History, 34(4), 595-619.

Vonnard, P. (2018). L'Europe dans le monde du football: Genèse et formation de l'UEFA (1930-1960). Bruselas: Peter Lang.

Vonnard, P. (2020). Gouverner le football européen: La prise d'indépendance de l'UEFA vis-à-vis du pouvoir de la FIFA (1953-1961). En M. Schotté y J. Vincent (dirs.), Le sport et ses pouvoirs (pp. 113-133). Limoges: PULIM.

Vonnard, P., y Quin, G. (2017). Did South America foster European football? Transnational influences on the continentalisation of FIFA and the creation of UEFA, 19261959. Sport in Society, 20(10), 1424-1439.

Vonnard, P., y Quin, G. (2019). Promouvoir et jouer au football pendant la guerre: La Fédération Internationale de Football Association: les forces de l'axe et la deuxième guerre mondiale. Hispania Nova, 17. Recuperado de https://e-revistas.uc3m.es/index.php/HISPNOV/article/view/4524.

Wahlig, H. (2010). Ein Tor zur Welt: Der deutsche Fußball und die FIFA 1945-1950. Göttingen: Verlag die Werksatt. 